\title{
Native pulmonary valve infective endocarditis with septic embolisms and an infra-annular abscess
}

\author{
Javier Rodriguez Lega ${ }^{1}$, Uxue Murgoitio Esandi ${ }^{1}$, and Angel Gonzalez Pinto ${ }^{1}$ \\ ${ }^{1}$ Gregorio Marañón General University Hospital Cardiac Surgery Service
}

December 4, 2020

\begin{abstract}
Infective endocarditis is nowadays one of the main indications for valvular surgery. When occurring in the right chambers of the heart, the tricuspid valve is the most commonly affected. Although this disease is usually present in injecting-drug abusers or patients with other predisposing risk factors, the incidence of native pulmonary valve infective endocarditis is the least frequent. This case report presents a 51-year-old patient with no relevant medical history that was admitted to our institution with sepsis. Further studies revealed a native pulmonary valve infective endocarditis complicated with infra-annular abscess and pulmonary septic embolisms. The following describes the clinical presentation of the case, echocardiograms, the surgical procedure, and the postoperative period. To date, few cases of native pulmonary valve infective endocarditis with no previous risk factors have been reported.
\end{abstract}

Title:

Native pulmonary valve infective endocarditis with septic embolisms and an infra-annular abscess.

Authors:

Rodríguez Lega, Javier ${ }^{1}$; Murgoitio Esandi, Uxue ${ }^{1}$; González Pinto, Ángel ${ }^{1}$.

Institution(s):

${ }^{1}$ Department of Cardiac Surgery, Gregorio Marañón General University Hospital, Madrid (Spain).

Correspondence:

Javier Rodríguez Lega, Department of Cardiovascular Surgery, Gregorio Marañón General University Hospital, Doctor Esquerdo St. 46, 28007, Madrid, Spain. Email: jrl2003@gmail.com, frlega@salud.madrid.org

Funding/conflict of interest: none.

The report of a case is exempt from approval by the ethics committee in our center provided that the patient expresses his consent to the publication of the case, which was obtained on 3 December, 2020.

\begin{abstract}
Infective endocarditis is nowadays one of the main indications for valvular surgery. When occurring in the right chambers of the heart, the tricuspid valve is the most commonly affected. Although this disease is usually present in injecting-drug abusers or patients with other predisposing risk factors, the incidence of native pulmonary valve infective endocarditis is the least frequent.
\end{abstract}


This case report presents a 51-year-old patient with no relevant medical history that was admitted to our institution with sepsis. Further studies revealed a native pulmonary valve infective endocarditis complicated with infra-annular abscess and pulmonary septic embolisms. The following describes the clinical presentation of the case, echocardiograms, the surgical procedure, and the postoperative period.

To date, few cases of native pulmonary valve infective endocarditis with no previous risk factors have been reported.

Keywords

cardiac surgery; infective endocarditis; native pulmonary valve.

Abbreviations and acronyms

Computer tomography: $\mathrm{CT}$

Infective endocarditis: IE

Pulmonary valve: PV

Pulmonary valve infective endocarditis: PVIE

Methicillin-sensitive Staphylococcus aureus: MSSA

Transthoracic echocardiogram: TTE

Transesophageal echocardiogram: TEE

Introduction

The incidence of infective endocarditis (IE) has increased over the last decades. Between January 2016 and March 2018, the European Infective Endocarditis Registry (Euro-Endo) ${ }^{2}$ recorded 3,116 adult patients with IE according to diagnostic criteria in 2015 of theEuropean Society of Cardiology (ESC) ${ }^{3}$.

IE was most frequent on native valves $(56.6 \%)$, followed by prosthetic valves $(30.1 \%)$ and, finally, on intracardiac devices $(9.9 \%)$. The majority of cases were community-acquired IE $(65.66 \%)$ and the most frequently isolated microorganism was Staphylococcus sp (44.1\%), followed by Enterococcus sp (15.8\%) and Streptococcus $s p(12.3 \%)$. The main complications were embolisms in $20.6 \%$ of the patients, which usually associated with right and aortic valve IE, even more frequently if caused by Staphylococcus aureus or vegetations existed.

Regarding location, left IE was more common than right IE. The aortic valve was the most frequently affected valve $(49.5 \%)$, then the mitral valve $(42 \%)$ and the tricuspid valve (11.4\%), being the pulmonary valve (PV) the least frequent with only $2.4 \%$ of the $\operatorname{cases}^{3,5}$.

Most often, pulmonary valve infective endocarditis (PVIE) occurs in active injecting-drug abusers or whenever chronic vascular accesses or pacemakers are present ${ }^{5}$. Other predisposing risk factors for native valve IE are immunosuppression, diabetes mellitus, cancer, kidney dialysis and HIV. The incidence for native PVIE is reckoned to be solely around 0.2 to $1.2 \%^{1}$.

An explanation of the clinical presentation, echocardiograms, the surgical procedure and postoperative care of an adult patient with native PVIE and no significant medical history nor risk factors is included.

Case report

A 51-year-old healthy male patient with no relevant medical history was admitted to the emergency room with a one-week history of fever having a mild response to paracetamol, headaches, myalgia and neck stiffness. He denied coughing, dyspnea or chest pain. During physical examination, his heart rate was 145 bpm and blood pressure was $90 / 60 \mathrm{mmHg}$. Cardiopulmonary auscultation was rhythmic and showed no abnormalities. Laboratory results on admission showed a leukocyte count of $22,860 / \mu \mathrm{L}$ with neutrophilia $(84.9 \%)$ and thrombocytopenia $(49,000 / \mu \mathrm{L})$. Acute kidney injury was also shown by a creatinine of 1.4 $\mathrm{mg} / \mathrm{dL}$ and hyponatremia $(126 \mathrm{mmol} / \mathrm{L})$. Bilirrubin was $1.4 \mathrm{mg} / \mathrm{dL}$. Arterial blood gases showed hypoxemia 
and hypocapnia with secondary respiratory alkalosis. Acute-phase reactants were elevated with a C-reactive protein of $23.4 \mathrm{mg} / \mathrm{dL}(0.0-0.5)$ and a procalcitonin of $5.26 \mu \mathrm{g} / \mathrm{L}(0.00-0.50)$. The chest X-Ray showed no significant findings. Blood, urine cultures and a nasal swab were drawn, which were all positive for Methicillin-sensitive Staphylococcus aureus (MSSA).

The patient was directly admitted into the intensive care unit with the diagnosis of septic shock. Broadspectrum antibiotic therapy was started with Meropenem and Vancomycin. Blood cultures were drawn again the next day, being still positive for MSSA. The patient required vasoactive drugs for 48 hours. After 72 hours, the patient was stable and transferred to the cardiology ward. Antibiotic therapy was then changed to Meropenem and Cloxacillin.

The transthoracic echocardiogram (TTE) showed no abnormalities. Transesophageal echocardiogram (TEE) was not performed due to the patient's rejection of this procedure. On the $6^{\text {th }}$ day of hospitalization, the patient asked to be discharged against medical advice. The antibiotic therapy was discontinued.

Two days later, the patient returned to the emergency department with persistent fever and signs of right heart failure in physical examination. The patient was then readmitted and antibiotic therapy with Daptomycin and Cloxacillin was started. The chest X-Ray showed bilateral mild pleural effusions and images suggesting bilateral multilobar pneumonic foci similar to pulmonary septic embolisms (Figure_1).

A new TTE was performed on suspicion of right heart IE, but showed no abnormal findings. TEE was then performed showing nodular images on the PV suggestive of large vegetations (Figure_2) causing substantial valve destructuration and severe pulmonary regurgitation (IV/IV) (Figure_3). Tricuspid regurgitation was mild without suggestive signs of IE. The rest of the study was normal.

The case was accepted for urgent surgery. Among the different techniques for the reconstruction of the pulmonary valve have been described, a biologic valve replacement according to Kogon's technique ${ }^{4}$ was the chosen procedure. On the surgical field, a severely unstructured PV with large vegetations anchored to the anterior and right leaflets was identified (Figure_4). There was also an infra-annular abscess, not visualized in the TEE, rightly below the left leaflet that spread through the posterior infundibular wall with no signs of perforation.

Once the vegetations were excised, the abscess was also drained, cleaned and finally closed with a bovine pericardial patch (Figure_5). The pulmonary valve was replaced with a bioprosthetic valve. The main pulmonary artery and right ventricular infundibulum were reconstructed with another bovine pericardial patch (Figure_6).

The intraoperative TEE showed no paravalvular leaks with a normal functioning prosthesis. The patient experienced an uncomplicated postoperative period, being extubated immediately after surgery and transferred to the ward 48 hours later.

A postoperative computed tomography scan showed images suggestive of bilateral pulmonary septic embolisms and thromboembolisms with infarcts (Figure_7) The microbiological test of the valve confirmed IE caused byStaphylococcus aureus .

The patient was discharged once the antibiotic therapy was completed and remains asymptomatic.

\section{Discussion}

The native PVIE is the least frequent presentation of IE. If IE is suspected, a TEE is recommended for its correct assessment. TTE lacks sensibility for PV disease evaluation, as the echocardiographic window is poor. Pulmonary septic embolisms are infrequent and are usually associated with right IE. To conclude, we can say that VP replacement is an easily-reproducible surgical technique in adults, even though PVIE is uncommon. 


\section{Figure Legends}

Figure_1. Chest X-ray showing images suggestive of pulmonary septic embolisms $(*)$.

Figure_2. TEE showing pulmonary valve large vegetations $\left(^{*}\right)$.

Figure_3. TEE showing severe pulmonary valve regurgitation.

Figure_4. Pulmonary valve affected by endocarditis (*: vegetations).

Figure_5. Pericardial patch closing the abscess $\left({ }^{*}\right)$.

Figure_6. Final result of the pulmonary artery pericardial patch. Ao, aorta; RA, right atrium; PA, reconstructed pulmonary artery.

Figure_7. Chest CT showing pulmonary embolisms $(*)$.

\section{References}

\begin{tabular}{ll}
\hline & Cabell CH, Abrutyn E, Fowler VG, Hoen B, Miro \\
JM, Corey GR, Olaison L, Pappas P, Anstrom \\
KJ, Stafford JA, Eykyn S, Habib G, Mestres CA, \\
Wang A; International Collaboration on \\
Endocarditis Merged Database (ICE-MD) Study \\
Group Investigators. Use of surgery in patients \\
with native valve infective endocarditis: results \\
from the International Collaboration on \\
Endocarditis Merged Database. Am Heart J 2005; \\
150: 1092-1098. \\
\hline Habib G, Erba PA, Iung B, Donal E, Cosyns B, \\
Laroche C, Popescu BA, Prendergast B, Tornos P, \\
Sadeghpour A, Oliver L, Vaskelyte JJ, Sow R, \\
Axler O, Maggioni A, Lancellotti P. Clinical \\
presentation, aetiology and outcome of infective \\
endocarditis. Results of the ESC-EORP \\
EURO-ENDO (European infective endocarditis) \\
registry: a prospective cohort study. Eur Heart J. \\
2019; 40: 3222-3232. \\
Habib G, Lancellotti P, Antunes MJ, Bongiorni \\
MG, Casalta JP, Del Zotti F, Dulgueru R, El \\
Khoury G, Erba PA, Iung B, M JM, Mulder BJ \\
Plnska-Gosciniak E, Price S, Roos-Hesselink J, \\
Snygg-Martin U, Thuny F, Tornos P, Vilacosta I, \\
Zamorano JL. The Task Force for the \\
Management of Infective Endocarditis of the \\
European Society of Cardiology (ESC). 2015 ESC \\
Guidelines for the management of infective \\
endocarditis. Eur Heart J. 2015; 36: 3075-3128. \\
(a)
\end{tabular}




\begin{tabular}{ll}
\hline & Cabell CH, Abrutyn E, Fowler VG, Hoen B, Miro \\
& JM, Corey GR, Olaison L, Pappas P, Anstrom \\
& KJ, Stafford JA, Eykyn S, Habib G, Mestres CA, \\
& Wang A; International Collaboration on \\
& Endocarditis Merged Database (ICE-MD) Study \\
& Group Investigators. Use of surgery in patients \\
& with native valve infective endocarditis: results \\
& from the International Collaboration on \\
& Endocarditis Merged Database. Am Heart J 2005; \\
& $150:$ 1092-1098. \\
\hline 4 & Kogon BE, Rodby KA, Kirshbom PM, Kanter KR, \\
& Lyle T, McConnell M, Book WM. Adult \\
& Congenital Pulmonary Valve Replacement: A \\
& Simple Effective, and Reproducible Technique. \\
& Congenit Heart Dis. 2007; 2: 314-318. \\
& https://www.ctsnet.org/article/adult-congenital- \\
& pulmonary-valve-replacement-simple-effective-and- \\
& reproducible-technique \\
& Liekiene D, Bezuska L, Semeniene P, Cypiene R, \\
& Lebetkevicius V, Tarutis V, Barysiene J, \\
& Rucinskas K, Sirvydis V. Surgical Treatment of \\
Infective Endocarditis in Pulmonary Position-15 & Years Single Centre Experience. Medicina \\
& (Kaunas). 2019; 55. pii: E608. \\
\hline
\end{tabular}

\section{Hosted file}

Figures with accompanying legend.pdf available at https://authorea.com/users/381282/articles/ 497095-native-pulmonary-valve-infective-endocarditis-with-septic-embolisms-and-an-infraannular-abscess 